\title{
Instability for the Navier-Stokes Equations on the 2-Dimensional Torus and a Lower Bound for the Hausdorff Dimension of Their Global Attractors
}

\section{Vincent Xiaosong Liu}

Department of Mathematics, University of Southern California, Los Angeles, CA 90089-1113, USA

Received May 21, 1991; in revised form October 11, 1991

Abstract. We prove instability of stationary solutions of the Navier-Stokes equations on the domain $[0,2 \pi] \times[0,2 \pi]$ with periodic boundary condition for a class of external forces for large Reynolds number. Moreover, we give a lower bound for the Hausdorff dimension of the global attractors.

\section{Introduction}

This paper is a continuation of our previous work [9]. We consider the two dimensional Navier-Stokes equations for a viscous incompressible fluid with spatially periodic boundary conditions (with periods $2 \pi, 2 \pi$ ). The Navier-Stokes equations with velocity $u$ and external force $f$ (assume $f$ is time independent) in functional form can be written as (see $[3,14,15])$

$$
\begin{aligned}
\frac{d u}{d t}+A u+B(u, u) & =f, \\
u(0) & =u_{0},
\end{aligned}
$$

in a Hilbert space $H$, where $H$ consists of those $u$ such that

$$
\begin{aligned}
u & =\sum_{j=\left(j_{1}, j_{2}\right) \in Z^{2}} u_{j} e^{i\left(j_{1} x_{1}+j_{2} x_{2}\right)}, \quad u_{j} \in C^{2}, \quad u_{-j}=\bar{u}_{j}, \quad u_{0}=0, \\
j * u_{j} & =0, \quad \text { for each } j \\
|u|^{2} & =(2 \pi)^{2} \sum_{j \in Z^{2}}\left|u_{j}\right|^{2}<\infty .
\end{aligned}
$$

Let $P$ be the orthogonal projection onto $H$ in $\left(L^{2}(\Omega)\right)^{2}$ (where $\Omega=[0,2 \pi]$ $\times[0,2 \pi])$, then

$$
\begin{gathered}
A u=-P \Delta u, \\
B(v, w)=P[(v * \nabla) w] .
\end{gathered}
$$


We denote $D(A)$ the domain of the operator $A$.

Now for $\left(k_{1}, k_{2}\right) \neq(0,0)$, we define

$$
\begin{aligned}
& W_{\left(k_{1}, k_{2}\right)}=\frac{1}{\sqrt{2} \pi \sqrt{k_{1}^{2}+k_{2}^{2}}}\left(\begin{array}{c}
k_{2} \\
-k_{1}
\end{array}\right) \cos \left(k_{1} x_{1}+k_{2} x_{2}\right), \\
& W_{\left(k_{1}, k_{2}\right)}^{\prime}=\frac{1}{\sqrt{2} \pi \sqrt{k_{1}^{2}+k_{2}^{2}}}\left(\begin{array}{c}
k_{2} \\
-k_{1}
\end{array}\right) \sin \left(k_{1} x_{1}+k_{2} x_{2}\right) .
\end{aligned}
$$

Let

$$
K=\left\{\left(k_{1}, k_{2}\right) \mid k_{1}>0 \text { or } k_{1}=0, k_{2}>0\right\} .
$$

We see that $W_{\left(k_{1}, k_{2}\right)}, W_{\left(k_{1}, k_{2}\right)}^{\prime},\left(k_{1}, k_{2}\right) \in K$ are eigenvectors of $A$ with eigenvalues $k_{1}^{2}+k_{2}^{2}$; and those $W_{\left(k_{1}, k_{2}\right)}, W_{\left(k_{1}, k_{2}\right)}^{\prime}$ form an orthonormal basis in $H$.

It is easy to see for $\left(k_{1}, k_{2}\right) \neq(0,0)$,

$$
\begin{aligned}
& P\left(\begin{array}{l}
\alpha \\
\beta
\end{array}\right) \cos \left(k_{1} x_{1}+k_{2} x_{2}\right)=\frac{\sqrt{2} \pi\left(\alpha k_{2}-\beta k_{1}\right)}{\sqrt{k_{1}^{2}+k_{2}^{2}}} W_{\left(k_{1}, k_{2}\right)}, \\
& P\left(\begin{array}{l}
\alpha \\
\beta
\end{array}\right) \sin \left(k_{1} x_{1}+k_{2} x_{2}\right)=\frac{\sqrt{2} \pi\left(\alpha k_{2}-\beta k_{1}\right)}{\sqrt{k_{1}^{2}+k_{2}^{2}}} W_{\left(k_{1}, k_{2}\right)}^{\prime},
\end{aligned}
$$

for any $\alpha, \beta$.

In this paper, we consider the Navier-Stokes equations with external forces $f_{0}=s^{2} \lambda W_{(0, s)}^{\prime}$. A corresponding stationary solution is

$$
u_{0}=\lambda W_{(0, s)}^{\prime},
$$

where $\lambda$ is a parameter. For simplicity, we only consider $\lambda>0$; the case $\lambda<0$ is the same.

As in $[5,15]$, the nondimensional Grashof number is defined by

$$
G=\frac{|f|}{v^{2} \lambda_{1}} .
$$

In our case here, since the viscosity $v=1$ and $\lambda_{1}=1$, so

$$
G=\left|f_{0}\right|=s^{2} \lambda \text {. }
$$

The Navier-Stokes equations linearized around $u_{0}$ are

$$
\frac{d \omega}{d t}+\Theta\left(u_{0}\right) w=0
$$

where

$$
\Theta\left(u_{0}\right) w=A w+B\left(w, u_{0}\right)+B\left(u_{0}, w\right) .
$$

We concentrate on the linearized Navier-Stokes equations (11). We show below that $\Theta\left(u_{0}\right)$ has negative eigenvalues for large $\lambda$ and instability of the corresponding Navier-Stokes equations follows from this $[2,12]$. We give an estimate to the dimension of these unstable directions at the stationary solution $u_{0}$ including the following results: 
- A positive answer to a problem of V. I. Arnold (see [1]): Is it true that the minimum of the Hausdorff dimension of minimal attractors of the Navier-Stokes equation (on, say, the two-dimensional torus) grows as the Reynolds number increases?

- An answer to a problem of M. I. Vishik (see [1]): Find a lower bound for the Hausdorff dimension of the attractor of the two-dimensional Navier-Stokes system of equations for large values of Reynolds number.

In Sect. 2, we will reduce the eigenvalue problem for $\Theta\left(u_{0}\right)$ to an infinite system (uncoupled) of three term recurrent relations. Section 3 will recall some properties of these recurrent relations. Section 4 gives some Lemmas and Sect. 5 proves our main results.

\section{Reduction of the Problem}

As in [9], we use a Fourier expansion, and consequently we reduce the eigenvalue problem for $\Theta\left(u_{0}\right)$ to a set of three term recurrent relations among the Fourier coefficients.

First, by the definitions of $B(u, v), P, W_{\left(k_{1}, k_{2}\right)}, W_{\left(k_{1}, k_{2}\right)}^{\prime}$ and using (6), (7), by direct calculations, we get

Lemma 1. For every $\left(k_{1}, k_{2}\right) \neq(0,0)$, we have:

(A).

$$
\begin{aligned}
& B\left(W_{\left(k_{1}, k_{2}\right)}, W_{(0, s)}^{\prime}\right)+B\left(W_{(0, s)}^{\prime}, W_{\left(k_{1}, k_{2}\right)}\right) \\
& \quad=\frac{k_{1}\left(-s^{2}+k_{1}^{2}+k_{2}^{2}\right)}{2 \sqrt{2} \pi \sqrt{k_{1}^{2}+k_{2}^{2}}}\left\{\frac{1}{\sqrt{k_{1}^{2}+\left(k_{2}+s\right)^{2}}} W_{\left(k_{1}, k_{2}+s\right)}-\frac{1}{\sqrt{k_{1}^{2}+\left(k_{2}-s\right)^{2}}} W_{\left(k_{1}, k_{2}-s\right)}\right\},
\end{aligned}
$$

and

(B).

$$
\begin{aligned}
& B\left(W_{\left(k_{1}, k_{2}\right)}^{\prime}, W_{(0, s)}^{\prime}\right)+B\left(W_{(0, s)}^{\prime}, W_{\left(k_{1}, k_{2}\right)}^{\prime}\right) \\
& \quad=\frac{k_{1}\left(-s^{2}+k_{1}^{2}+k_{2}^{2}\right)}{2 \sqrt{2} \pi \sqrt{k_{1}^{2}+k_{2}^{2}}}\left\{\frac{1}{\sqrt{k_{1}^{2}+\left(k_{2}+s\right)^{2}}} W_{\left(k_{1}, k_{2}+s\right)}^{\prime}-\frac{1}{\sqrt{k_{1}^{2}+\left(k_{2}-s\right)^{2}}} W_{\left(k_{1}, k_{2}-s\right)}^{\prime}\right\} .
\end{aligned}
$$

In the above equations, we notice that the denominators of some terms could be zero. But from the process of getting these formulas, we see that those terms whose denominators are zero are also zero.

We want to find an eigenvector $V$ and a number $\sigma, \operatorname{Re} \sigma \geqq 0$, such that

$$
\Theta\left(u_{0}\right) V=-\sigma V
$$

We decompose the eigenvector $V$ as even and odd part:

$$
\begin{aligned}
V & =W+W^{\prime}, \\
W & =\sum_{\left(k_{1}, k_{2}\right) \in K} a_{\left(k_{1}, k_{2}\right)} W_{\left(k_{1}, k_{2}\right)}, \\
W^{\prime} & =\sum_{\left(k_{1}, k_{2}\right) \in K} a_{\left(k_{1}, k_{2}\right)}^{\prime} W_{\left(k_{1}, k_{2}\right)}^{\prime} .
\end{aligned}
$$


From Lemma 1 and the properties of operators $A$ and $B$, we can decompose the above Eq. (13) into the equations

$$
\begin{gathered}
\Theta\left(u_{0}\right) W=-\sigma W, \\
\Theta\left(u_{0}\right) W^{\prime}=-\sigma W^{\prime} .
\end{gathered}
$$

We note (17) and (18) are the same. We concentrate on Eq. (17). Equation (18) can be handled analogously.

From Lemma 1, we obtain

$$
\begin{aligned}
B\left(W, u_{0}\right)+B\left(u_{0}, W\right)= & \lambda B\left(W, W_{(0, s)}^{\prime}\right)+\lambda B\left(W_{(0, s)}^{\prime}, W\right) \\
= & \lambda \sum_{\left(k_{1}, k_{2}\right) \in K} a_{\left(k_{1}, k_{2}\right)}\left\{B\left(W_{\left(k_{1}, k_{2}\right)}, W_{(0, s)}^{\prime}\right)+B\left(W_{(0, s)}^{\prime}, W_{\left(k_{1}, k_{2}\right)}\right)\right\} \\
= & \lambda \sum_{\left(k_{1}, k_{2}\right) \in K} a_{\left(k_{1}, k_{2}\right)} \frac{k_{1}\left(k_{1}^{2}+k_{2}^{2}-s^{2}\right)}{2 \sqrt{2} \pi \sqrt{k_{1}^{2}+k_{2}^{2}}}\left\{\frac{1}{\sqrt{k_{1}^{2}+\left(k_{2}+s\right)^{2}}} W_{\left(k_{1}, k_{2}+s\right)}\right. \\
& \left.-\frac{1}{\sqrt{k_{1}^{2}+\left(k_{2}-s\right)^{2}}} W_{\left(k_{1}, k_{2}-s\right)}\right\} .
\end{aligned}
$$

Now, we substitute $W$ and (19) into (17), and compare the coefficients of $W_{\left(k_{1}, k_{2}\right)}$. We get the following recurrent relations for $a_{\left(k_{1}, k_{2}\right)}$ :

$$
\begin{aligned}
& \left(k_{1}^{2}+k_{2}^{2}+\sigma\right) a_{\left(k_{1}, k_{2}\right)}+\frac{\lambda k_{1}\left(k_{1}^{2}+\left(k_{2}-s\right)^{2}-s^{2}\right)}{2 \sqrt{2} \pi \sqrt{k_{1}^{2}+\left(k_{2}-s\right)^{2}} \sqrt{k_{1}^{2}+k_{2}^{2}}} a_{\left(k_{1}, k_{2}-s\right)} \\
& -\frac{\lambda k_{1}\left(k_{1}^{2}+\left(k_{2}+s\right)^{2}-s^{2}\right)}{2 \sqrt{2} \pi \sqrt{k_{1}^{2}+\left(k_{2}+s\right)^{2}} \sqrt{k_{1}^{2}+k_{2}^{2}}} a_{\left(k_{1}, k_{2}+s\right)}=0, \quad \text { for }\left(k_{1}, k_{2}\right) \in K .
\end{aligned}
$$

For each fixed $k_{1} \geqq 0$, the above equation gives a three term recurrent relation among $a_{\left(k_{1}, k_{2}-s\right)}, a_{\left(k_{1}, k_{2}\right)}, a_{\left(k_{1}, k_{2}+s\right)}$. So the problem of solving (17) is reduced to solving (20) for each fixed $k_{1}$. It is easy to note that for $k_{1}=0$, under the assumption $\operatorname{Re} \sigma \geqq 0$, the only solution of $(20)$ is $a_{\left(0, k_{2}\right)}=0, \forall k_{2}>0$. So we assume $k_{1}>0$ below.

From (20), we get

Lemma 2. For every fixed $k_{1}>0$,

$$
c_{\left(k_{1}, k_{2}\right)} b_{\left(k_{1}, k_{2}\right)}+b_{\left(k_{1}, k_{2}-s\right)}-b_{\left(k_{1}, k_{2}+s\right)}=0
$$

where

$$
\begin{aligned}
b_{\left(k_{1}, k_{2}\right)} & =\frac{k_{1}^{2}+k_{2}^{2}-s^{2}}{\sqrt{k_{1}^{2}+k_{2}^{2}}} a_{\left(k_{1}, k_{2}\right)}, \\
c_{\left(k_{1}, k_{2}\right)} & =\frac{2 \sqrt{2} \pi\left(k_{1}^{2}+k_{2}^{2}\right)\left(k_{1}^{2}+k_{2}^{2}+\sigma\right)}{\lambda k_{1}\left(k_{1}^{2}+k_{2}^{2}-s^{2}\right)} .
\end{aligned}
$$

The problem of solving (20) now becomes solving (21) under the assumption $\operatorname{Re} \sigma \geqq 0$. Because the eigenvector $W$ belongs to the space $D(A)$, we try to find nontrivial solutions of (21) such that

$$
b_{\left(k_{1}, k_{2}\right)} \rightarrow 0 \quad \text { if } \quad\left|k_{2}\right| \rightarrow \infty .
$$


If the above is true, then from (21), for all $n>0$,

$$
\left|k_{2}\right|^{n} b_{\left(k_{1}, k_{2}\right)} \rightarrow 0 \quad \text { if } \quad\left|k_{2}\right| \rightarrow \infty .
$$

Now we let

$$
a_{n}=c_{(t, s n+r)}=\frac{2 \sqrt{2} \pi\left(t^{2}+(s n+r)^{2}\right)\left(t^{2}+(s n+r)^{2}+\sigma\right)}{\lambda t\left(t^{2}+(s n+r)^{2}-s^{2}\right)},
$$

where $t$ is a positive integer, $n$ is an integer and $r=1, \ldots, s-1$. And we let

$$
c_{n}=b_{(t, s n+r)}=\frac{t^{2}+(s n+r)^{2}-s^{2}}{\sqrt{t^{2}+(s n+r)^{2}}} a_{(t, s n+r)} .
$$

From Lemma 2, for each fixed positive integer $t$ and fixed $r=1, \ldots, s-1$, we get the following three term recurrent relation

$$
a_{n} c_{n}+c_{n-1}-c_{n+1}=0, \quad n=0, \pm 1, \pm 2, \ldots
$$

By the trivial solution of (27) we mean the solution $c_{n}=0$ for $\forall n$. We want to find nontrivial solutions of the above Eq. (27) such that

$$
\begin{aligned}
\operatorname{Re} \sigma & \geqq 0, \\
\lim _{|n| \rightarrow \infty} \operatorname{Re} c_{n} & =0 .
\end{aligned}
$$

In the following, by the nontrivial solutions of (27) we mean those nontrivial solutions of (27) that also satisfy the conditions (28) and (29).

\section{The Three Term Recurrent Relations and Continued Fractions}

We first recall some results $[9,11,16]$. For the property of continued fractions, we refer to [8]. We consider the three term recurrent relations:

$$
a_{n} c_{n}+c_{n-1}-c_{n+1}=0,
$$

where $a_{n}, c_{n}$ are complex numbers and $n=0, \pm 1, \pm 2, \ldots$ We have

Theorem 1. (cf. $[9,11,16]$ ) Assume

$$
\begin{aligned}
\operatorname{Re} a_{n} & >0, \quad \text { for } \forall n \neq 0,1, \\
\lim _{|n| \rightarrow \infty} \operatorname{Re} a_{n} & =\infty .
\end{aligned}
$$

Then the following two conditions are equivalent:

(A). There exists a non-trivial solution $\left\{c_{n}\right\}$ of (30) such that

$$
\lim _{|n| \rightarrow \infty} c_{n}=0
$$

(B). The following equation is true.

$$
a_{0}+\frac{1}{a_{-1}+\frac{1}{a_{-2}+\cdots}}=\frac{-1}{a_{1}+\frac{1}{a_{2}+\cdots}}
$$


Moreover, the solution which satisfies the condition (A) is unique within a constant factor.

Corollary 1. Under the conditions (31), (32). If

$$
a_{k}=a_{-k}, \quad \text { for } \forall k \neq 0,
$$

then condition $(A)$ of Theorem 1 is equivalent to

$$
-\frac{a_{0}}{2}=\frac{1}{a_{1}+\frac{1}{a_{2}+\cdots}} .
$$

Corollary 2. Under the conditions (31), (32), if

$$
a_{k}=a_{-(k-1)}, \quad \text { for } \forall k,
$$

then condition (A) of Theorem 1 is equivalent to

$$
a_{1}+\frac{1}{a_{2}+\frac{1}{a_{3}+\cdots}}=i \text { or }-i \text {. }
$$

\section{Some Lemmas}

Now, we give the following lemmas.

Lemma 3. Assume $t \geqq s$. Then for each fixed $t$ and $r$, Eq. (27) has only the trivial solution.

Proof. First, assume $t>s$ or $t=s, r \neq 0$. From (25)

$$
\begin{aligned}
& \operatorname{Re} a_{n}>0, \text { for } \forall n, \\
& \lim _{|n| \rightarrow \infty} \operatorname{Re} a_{n}=\infty .
\end{aligned}
$$

Now it is easy to see that (34) is impossible, so by Theorem $1,(27)$ has only the trivial solution.

Second, assume $t=s, r=0$. From (25) and (26),

$$
\begin{gathered}
\operatorname{Re} a_{n}>0, \text { for } \forall n \neq 0, \\
\lim _{|n| \rightarrow \infty} \operatorname{Re} a_{n}=\infty, \\
c_{0}=0 .
\end{gathered}
$$

By the proof of Theorem 1 (see, for example, [9]), we see that if (27) has nontrivial solution, then

$$
c_{n} \neq 0, \quad \text { for } \forall n,
$$

which is a contradiction with $c_{0}=0$.

Denote by $[x]$ the largest integer less than or equal to $x$. 
Lemma 4. 'Assume $r=0$. For each $t=1, \ldots, s-1$, there is a unique $\lambda_{0}(t)>0$ which increases monotonically with $t$ such that

- If $\lambda \geqq \lambda_{0}(t)$, then there is a nontrivial solution of $(27)$ with $\sigma=\sigma_{t}(\lambda)$.

- If $0<\lambda<\lambda_{0}(t)$, then (27) has only the trivial solution.

Also $\sigma_{t}(\lambda)$ increases monotonically with $\lambda \geqq \lambda_{0}(t)$ and $\sigma_{t}\left(\lambda_{0}(t)\right)=0$,

$$
\sigma_{t}(\lambda) \leqq O(\lambda) \quad \text { if } \quad \lambda \rightarrow \infty .
$$

Moreover, if we assume $t=1,2, \ldots,[\sqrt{2 / 3} \mathrm{~s}]$, then

$$
\sigma_{t}(\lambda)=O(\lambda) \text { if } \lambda \rightarrow \infty .
$$

Proof. The proof is similar to the proof of Lemma 4 in [9].

Step 1. Since $r=0$, from (25)

$$
a_{n}=\frac{2 \sqrt{2} \pi\left(t^{2}+(s n)^{2}\right)\left(t^{2}+(s n)^{2}+\sigma\right)}{\lambda t\left(t^{2}+(s n)^{2}-s^{2}\right)},
$$

we see $a_{n}=a_{-n}$ and $\operatorname{Re} a_{n}>0$ for $\forall n \neq 0$. By Corollary 1, there is a nontrivial solution of (27) iff

$$
-\frac{a_{0}}{2}=\frac{1}{a_{1}+\frac{1}{a_{2}+\cdots}}
$$

Now we define the functions

$$
\begin{gathered}
f(\sigma)=\frac{1}{a_{1}+\frac{1}{a_{2}+\cdots},} \\
g(\sigma)=-\frac{a_{0}}{2}=\frac{\sqrt{2} \pi t\left(t^{2}+\sigma\right)}{\lambda\left(s^{2}-t^{2}\right)} .
\end{gathered}
$$

So (27) has a nontrivial solution iff

$$
f(\sigma)=g(\sigma) .
$$

Step 2. If (38) has a solution $\sigma$ with $\operatorname{Re} \sigma \geqq 0$, then $\sigma$ must be a real number.

This is because if $\operatorname{Im} \sigma \geqq 0$, then

$$
\arg \left(-a_{0}\right) \geqq \arg \left(a_{1}\right) \geqq \arg \left(a_{2}\right) \geqq \cdots
$$

so

$$
|\arg (f(\sigma))| \leqq \arg \left(a_{1}\right) \leqq \arg \left(-a_{0}\right) .
$$

But by (38) and the definition of the function $g$,

so

$$
|\arg (f(\sigma))|=|\arg (g(\sigma))|=\arg \left(-a_{0}\right),
$$

$$
\arg \left(a_{1}\right)=\arg \left(-a_{0}\right),
$$

we get $\operatorname{Im} \sigma=0$. The same consideration for the case $\operatorname{Im} \sigma \leqq 0$.

In the following, we restrict $\sigma$ such that $\sigma$ is real and $\sigma \geqq 0$. 
Step 3. From (38) and the definition of $g$, we obtain that (38) is equivalent to

$$
\left(t^{2}+\sigma\right)^{-1} f(\sigma)=\frac{\sqrt{2} \pi}{\lambda} \frac{t}{s^{2}-t^{2}} .
$$

By the definition of $f$, we have

$$
\left(t^{2}+\sigma\right)^{-1} f(\sigma)=\frac{1}{\left(t^{2}+\sigma\right) a_{1}+\frac{1}{\left(t^{2}+\sigma\right)^{-1} a_{2}+\cdots}},
$$

since $\left(t^{2}+\sigma\right) a_{2 n+1}$ increases monotonically and $\left(t^{2}+\sigma\right)^{-1} a_{2 n}$ decreases monotonically for $\sigma \geqq 0$, the function $\left(t^{2}+\sigma\right)^{-1} f(\sigma)$ decreases monotonically for $\sigma \geqq 0$. By a property of continued fractions, we know

$$
\left(t^{2}+\sigma\right)^{-1} f(\sigma)<\frac{1}{\left(t^{2}+\sigma\right) a_{1}}=\frac{\lambda t^{3}}{2 \sqrt{2} \pi\left(t^{2}+s^{2}\right)\left(t^{2}+s^{2}+\sigma\right)\left(t^{2}+\sigma\right)},
$$

so

$$
\left(t^{2}+\sigma\right)^{-1} f(\sigma)<\frac{\sqrt{2} \pi}{\lambda} \frac{t}{s^{2}-t^{2}}, \text { for } \sigma \text { large. }
$$

We want to find a $\lambda_{0}$ such that

$$
\left(t^{2}+0\right)^{-1} f(0)=\frac{\sqrt{2 \pi} t}{\lambda_{0}\left(s^{2}-t^{2}\right)}
$$

that is

$$
\lambda_{0} t^{-2} f(0)=\frac{\sqrt{2} \pi t}{s^{2}-t^{2}}
$$

Since

$$
\lambda t^{-2} f(0)=\frac{1}{\lambda^{-1} t^{2} a_{1}(0)+\frac{1}{\lambda t^{-2} a_{2}(0)+\cdots}}
$$

because $\lambda^{-1} t^{2} a_{2 n+1}(0)$ decreases monotonically with $\lambda>0$ and $\lambda t^{-2} a_{2 n}(0)$ is independent of $\lambda$, so the function $\lambda t^{-2} f(0)$ increases monotonically for $\lambda>0$. From

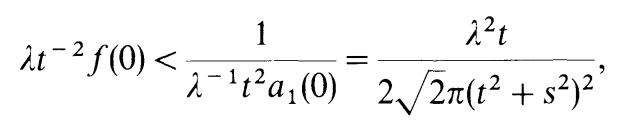

we get

$$
\lim _{\lambda \rightarrow 0} \lambda t^{-2} f(0) \rightarrow 0
$$

From

$$
\lambda t^{-2} f(0)>\frac{1}{\lambda^{-1} t^{2} a_{1}(0)+\frac{1}{\lambda t^{-2} a_{2}(0)}},
$$


so

$$
\lambda t^{-2} f(0)>\frac{\lambda}{t^{2} a_{1}(0)+\frac{1}{t^{-2} a_{2}(0)}} .
$$

We obtain

$$
\lim _{\lambda \rightarrow \infty} \lambda t^{-2} f(0) \rightarrow \infty
$$

Now from (44) and (45), since $\lambda t^{-2} f(0)$ increases monotonically for $\lambda>0$, there is a unique $\lambda_{0}>0$ such that (43) is true. So (42) is true. And we take this $\lambda_{0}$ as our $\lambda_{0}(t)$.

By Eq. (42), because $\lambda t^{-2} f(0)$ increases monotonically for $\lambda>0$, for $\forall \lambda>\lambda_{0}(t)$, we have

so

$$
\left(t^{2}+0\right)^{-1} f(0)>\frac{\sqrt{2} \pi}{\lambda} \frac{t}{s^{2}-t^{2}},
$$

$$
\left(t^{2}+\sigma\right)^{-1} f(\sigma)>\frac{\sqrt{2} \pi}{\lambda} \frac{t}{s^{2}-t^{2}}, \quad \text { for } \sigma \text { small. }
$$

From (41) and (46), we obtain that for $\forall \lambda>\lambda_{0}(t)$, there is a $\sigma>0$ such that (39) is true. Because $\left(t^{2}+\sigma\right)^{-1} f(\sigma)$ decreases monotonically for $\sigma \geqq 0$, we conclude that for each $\lambda>\lambda_{0}(t)$, there is a unique $\sigma_{t}(\lambda)>0$ which solves (39).

And for $\lambda=\lambda_{0}(t), \sigma_{t}(\lambda)=0$ solves (42), so solves (39).

And if $0<\lambda<\lambda_{0}(t)$, then

$$
\left(t^{2}+0\right)^{-1} f(0)<\frac{\sqrt{2} \pi}{\lambda} \frac{t}{s^{2}-t^{2}} .
$$

Since $\left(t^{2}+\sigma\right)^{-1} f(\sigma)$ decreases monotonically for $\sigma \geqq 0$, so (39) has no solutions with $\operatorname{Re} \sigma \geqq 0$.

Step 4. From (39) and (40), we get

$$
\frac{1}{\lambda^{-1}\left(t^{2}+\sigma\right) a_{1}+\frac{1}{\lambda\left(t^{2}+\sigma\right)^{-1} a_{2}+\cdots}}=\frac{\sqrt{2} \pi t}{s^{2}-t^{2}} .
$$

By the above inequality, we imply $\sigma_{t}(\lambda)$ increases monotonically in $\left[\lambda_{0}(t), \infty\right)$. Otherwise, from $\lambda^{-1}\left(t^{2}+\sigma\right) a_{2 n+1}$ decreases monotonically and $\lambda\left(t^{2}+\sigma\right)^{-1} a_{2 n}$ increases monotonically for $\lambda>0$, so the left-hand side of the above equation increases monotonically for some interval of $\lambda>0$ which is a contradiction.

Step 5. Because:

$$
\lambda_{0}(t) t^{-2} f(0)=\frac{\sqrt{2} \pi t}{s^{2}-t^{2}},
$$

so

$$
\frac{1}{\lambda_{0}(t)^{-1} t^{3} a_{1}(0)+\frac{1}{\lambda_{0}(t) t^{-3} a_{2}(0)+\cdots}}=\frac{\sqrt{2} \pi}{s^{2}-t^{2}} .
$$


From the above equality, we have that $\lambda_{0}(t)$ increases monotonically for $t=1, \ldots, s-1$. Otherwise $\lambda_{0}(t)^{-1} t^{3} a_{2 n+1}(0)$, will increase monotonically and $\lambda_{0}(t) t^{-3} a_{2 n}(0)$ will decrease monotonically, so the left-hand side of the above equation decreases monotonically for some interval of $\lambda>0$ which is a contradiction.

Step 6. From (38) and

$$
f(\sigma)<\frac{1}{a_{1}}
$$

we obtain

$$
\frac{\sqrt{2} \pi t\left(t^{2}+\sigma\right)}{\lambda\left(s^{2}-t^{2}\right)}<\frac{\lambda t^{3}}{2 \sqrt{2} \pi\left(t^{2}+s^{2}\right)\left(t^{2}+s^{2}+\sigma\right)}
$$

So

$$
\left(\sigma+t^{2}\right)\left(\sigma+t^{2}+s^{2}\right)<\frac{\lambda^{2} t^{2}\left(s^{2}-t^{2}\right)}{4 \pi^{2}\left(s^{2}+t^{2}\right)}
$$

and we get

$$
\sigma_{t}(\lambda) \leqq O(\lambda) \quad \text { if } \quad \lambda \rightarrow \infty
$$

From (38) and

$$
f(\sigma)>\frac{1}{a_{1}+\frac{1}{a^{2}}}
$$

we obtain

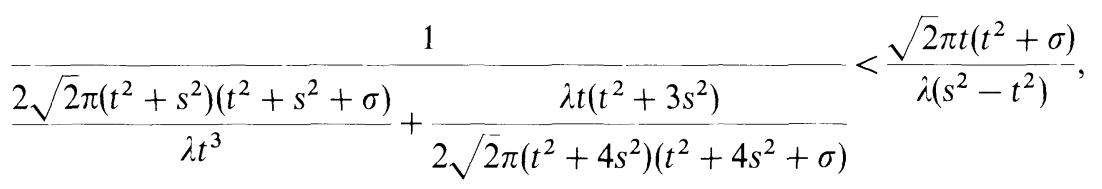

hence

$$
1<\frac{4 \pi^{2}\left(s^{2}+t^{2}\right)\left(s^{2}+t^{2}+\sigma\right)\left(t^{2}+\sigma\right)}{\lambda^{2} t^{2}\left(s^{2}-t^{2}\right)}+\frac{t^{2}\left(t^{2}+3 s^{2}\right)\left(t^{2}+\sigma\right)}{2\left(t^{2}+4 s^{2}\right)\left(t^{2}+4 s^{2}+\sigma\right)\left(s^{2}-t^{2}\right)},
$$

so

$$
\left(\sigma+t^{2}\right)\left(\sigma+t^{2}+s^{2}\right)>\frac{\lambda^{2} t^{2}\left(s^{2}-t^{2}\right)}{4 \pi^{2}\left(t^{2}+s^{2}\right)}\left\{1-\frac{t^{2}\left(t^{2}+3 s^{2}\right)\left(t^{2}+\sigma\right)}{2\left(t^{2}+4 s^{2}\right)\left(t^{2}+4 s^{2}+\sigma\right)\left(s^{2}-t^{2}\right)}\right\} .
$$

From the above (48), we see that if

$$
\frac{t^{2}\left(t^{2}+3 s^{2}\right)\left(t^{2}+\sigma\right)}{2\left(t^{2}+4 s^{2}\right)\left(t^{2}+4 s^{2}+\sigma\right)\left(s^{2}-t^{2}\right)}<1,
$$

then combining (48) with (47) we get

$$
\sigma_{t}(\lambda)=O(\lambda) \text { if } \quad \lambda \rightarrow \infty
$$


Now, we assume $1 \leqq t \leqq \sqrt{2 / 3} s$. We have

$$
\begin{aligned}
\frac{t^{2}\left(t^{2}+3 s^{2}\right)\left(t^{2}+\sigma\right)}{2\left(t^{2}+4 s^{2}\right)\left(t^{2}+4 s^{2}+\sigma\right)\left(s^{2}-t^{2}\right)} & <\frac{t^{2}\left(t^{2}+3 s^{2}\right)}{2\left(t^{2}+4 s^{2}\right)\left(s^{2}-t^{2}\right)} \\
& <\frac{t^{2}}{2\left(s^{2}-t^{2}\right)} \\
& \leqq 1 .
\end{aligned}
$$

We have proved Lemma 4.

\section{Instability and a Lower Bound for the Global Attractors}

Now we give our main result. The notations are the same as in the previous sections.

Theorem 2. Assume $1 \leqq t<s$. Then for each

$$
\lambda>\lambda_{0}([t])
$$

$\Theta\left(u_{0}\right)$ has at least $[t]$ negative eigenvalues $\eta_{j}, j=1, \ldots,[t]$; each of them has at least two eigenvectors. Also each $\eta_{j}(j=1, \ldots,[t])$ decreases monotonically with $\lambda \geqq \lambda_{0}([t])$ and

$$
-\eta_{j}(\lambda) \leqq O(\lambda) \text { if } \lambda \rightarrow \infty .
$$

Moreover, if $1 \leqq t \leqq[\sqrt{2 / 3}$ s $]$, then

$$
-\eta_{j}(\lambda)=O(\lambda) \quad \text { if } \lambda \rightarrow \infty, \quad \forall j=1, \ldots,[t] .
$$

Proof. By Lemma 4, since $\lambda_{0}(t)$ increases monotonically, so for $\forall \lambda>\lambda_{0}([t]), \Theta\left(u_{0}\right)$ has at least $[t]$ negative eigenvalues

$$
\eta_{j}(\lambda)=-\sigma_{j}(\lambda), \quad j=1, \ldots,[t] .
$$

Now from (17) and (18), we imply each of these eigenvalues has at least two eigenvectors. The other parts of the proof follow from Lemma 4.

Now we want to give an estimate of $\lambda_{0}(t)$. This can be done from the Step 6 of the proof Lemma $4 . \lambda_{0}(t)$ is determined by (43), that is

$$
f(0)=\frac{\sqrt{2} \pi t^{3}}{\lambda_{0}(t)\left(s^{2}-t^{2}\right)} .
$$

Now in Step 6 of the proof of Lemma 4, we let $\sigma=0$. From (47), we get

$$
\lambda_{0}(t)>\frac{2 \pi\left(s^{2}+t^{2}\right)}{\sqrt{s^{2}-t^{2}}} .
$$

From (48), we get

$$
\lambda_{0}(t)<\frac{2 \pi\left(t^{2}+s^{2}\right)}{\sqrt{s^{2}-t^{2}}} \frac{1}{\sqrt{1-\frac{\left(t^{2}+3 s^{2}\right) t^{4}}{2\left(t^{2}+4 s^{2}\right)^{2}\left(s^{2}-t^{2}\right)}}} .
$$


So $\lambda_{0}(t)$ is bounded by the inequalities (51) and (52). Now we let

$$
t^{\prime}=\left[\frac{s}{2}\right] \text {. }
$$

From (51) and (52), we easily obtain

$$
\frac{5 \pi}{\sqrt{3}} s<\lambda_{0}\left(t^{\prime}\right)<\frac{5 \sqrt{578} \pi}{\sqrt{1721}} s .
$$

Now we choose

where

$$
f_{0}=s^{2} \lambda^{\prime} W_{(0, s)}^{\prime}
$$

$$
\lambda^{\prime}=\frac{5 \sqrt{578} \pi}{\sqrt{1721}} S .
$$

From (10), we have that the Grashof number for the Navier-Stokes equations with the forcing term $f_{0}$ of $(54)$ is given by

$$
G=\frac{5 \sqrt{578} \pi}{\sqrt{1721}} s^{3} .
$$

By Theorem 2 and a result of [2], we see that for this forcing term $f_{0}$, the Hausdorff dimension of the global attractor $X$ is at least $2\left[\frac{s}{2}\right] \cong s$. So we obtain

Theorem 3. For the choices of external forces given by (54), we have

$$
\operatorname{dim}_{H}(X) \geqq c G^{1 / 3},
$$

where

$$
c=\left(\frac{\sqrt{1721}}{5 \sqrt{578} \pi}\right)^{1 / 3} .
$$

Remark. The above Theorem 3 gives an example that the Hausdorff dimension of the global attractor (minimal attractor) grows as the Reynolds number increases. Depending on how one understand Arnold's problem which is mentioned in the Introduction, we think that the above Theorem 3 gives a positive answer to his problem. We note that generally speaking, the answer to Arnold's problem is negative, because there are examples of Navier-Stokes equations on a torus with arbitrary big Reynolds number but its dynamics is trivial (cf. [10]). The above Theorem 3 also serves an answer to a problem of Vishik which also is mentioned in the Introduction.

Remark. The estimate of (55) could be improved by more carefully examining Eq. (27) since actually here we only considered a subset of those unstable directions at the stationary solution $u_{0}$. We will consider elsewhere more general forcing terms of the form $\lambda W_{\left(k_{1}, k_{2}\right)}$ and $\lambda W_{\left(k_{1}, k_{2}\right)}^{\prime}$. Moreover, we have already found points of stationary bifurcation for those cases (to be given elsewhere), and we hope we may find a Hopf bifurcation as well. 
We note that for the 2D Navier-Stokes equations with periods $L, 2 \pi$ and with forcing term $f_{0}=\left(\lambda g\left(x_{2}\right), 0\right)$, where $g\left(x_{2}\right)$ is a $2 \pi$-periodic function whose average value over the period is zero; if $L$ is big enough, instability for large Reynolds number has been shown by Babin and Vishik [2]. Also lower bounds for the Hausdorff dimension of the global attractor for the Geometry $[0, L] \times[0,2 \pi]$ where $L$ is big enough have been given in $[2,5,7]$.

An upper bound for the Hausdorff dimension of the global attractor has been given (cf. $[3,5,15])$ :

$$
\operatorname{dim}_{H}(X) \leqq c_{1} G^{2 / 3}(1+\log G)^{1 / 3},
$$

where $c_{1}$ is a nondimensional constant. For the Geometry $[0, L] \times[0,2 \pi]$ where $L$ is big enough, lower bound in the form

$$
\operatorname{dim}_{H}(X) \geqq c_{1} G^{2 / 3},
$$

has been given in [5]. Here we caution that the $G$ in the above equation (58) is different from the $G$ in Eq. (57) which is defined by Eq. (9). If one uses the same $G$ as in (57), then [5] can only get lower bound in the form

$$
\operatorname{dim}_{H}(X) \geqq c_{1} G^{2 / 5},
$$

which is not logarithmically close to the upper bound of (57) as stated in [5]. The same caution should apply to the results in [7] also.

Remark. After the completion of this paper, Titi told us that Foias and he (cf. [6]) have found a simple proof for the problems of Arnold and Vishik. Their proof is based on the work of Babin and Vishik [2] and by the observation that a periodic function with periods $\pi$ and $2 \pi$ is also a periodic function with periods $2 \pi$ and $2 \pi$. One can also check this by noting the specific form of eigenfunctions of the operator $\Theta\left(u_{0}\right)$ we get in this paper.

Based on the work of Babin and Vishik [2], if one uses the idea of Foias and Titi $[6]$ to transform the geometry $[0, L] \times[0,2 \pi]$ to the geometry $[0,2 \pi] \times[0,2 \pi]$, then one will get a lower bound exactly in the form given by Theorem 3 , of course with a different constant. Since the dynamics considered by Babin and Vishik [2] is only part of the dynamics on a square torus, we believe our method will reveal more information about the dynamics of the Navier-Stokes equations on the torus $[0,2 \pi] \times[0,2 \pi]$. The exploration will be pursued elsewhere.

Acknowledgements. I thank my advisor Professor N. D. Kazarinoff for valuable advice and helpful suggestions. And I thank Professor S. Smale for inspiration and valuable comments. Also I thank Professor E. Titi for helpful discussions.

\section{References}

1. Arnold, V. I. et al.: Some unsolved problems in the theory of differential equations and mathematics physics. Russ. Math. Surv. 44:4, 157-171 (1989)

2. Babin, A. V., Vishik, M. I.: Attractors of partial differential evolution equations and estimates of their dimension. Russ. Math. Surv. 38, 151-213 (1983)

3. Constantin, P., Foias, C.: Navier-Stokes equations. Chicago, IL: The University of Chicago Press 1988

4. Constantin, P., Foias, C., Temam, R.: On the large time Galerkin approximation of the Navier-Stokes equations. SIAM J. Number. Anal. 21, (4), 615-634 (1984) 
5. Constantin, P., Foias, C., Temam, R.: On the dimension of the attractors in two-dimensional turbulence. Physica D 30, 284-296 (1988)

6. Foias, C., Titi, E.: Private communications

7. Ghidaglia, J-M., Temam, R.: Lower bound on the dimension of the attractor for the Navier-Stokes equations in space dimension 3. In: Mechanics, Analysis and Geometry: 200 Years after Lagrange, Francaviglia, M., Holm, D. (eds.). Amsterdam: Elsevier

8. Jones, W. B., Thron, W. J.: Continued fractions, analytic theory and applications. Encyclopedia Math. Its Appl. Vol. 11 (1980)

9. Liu, V. X.: An example of instability for the Navier-Stokes equations on the 2-dimensional torus. Submitted to Comm. P.D.E.

10. Marchioro, C.: An example of absence of turbulence for any Reynolds number. Commun. Math. Phys. 105, 99-106 (1986)

11. Meshalkin, L. D., Sinai, Ya. G.: Investigation of the stability of a stationary solution of a system of equations for the plane movement of an incompressible viscous fluid. J. Appl. Math. Mech. 25 (1961)

12. Sattinger, D. H.: The mathematical problem of hydrodynamic stability. J. Math. Mech. 19, (9) (1970)

13. Smale, S.: Dynamics retrospective: Great problems, attempts that fialed. For "Non-linear Science: The Next Decade." Los Alamos, May 1990

14. Temam, R.: Navier-Stokes equations and nonlinear functional analysis. SIAM, Philadelphia, 1983

15. Temam, R.: Infinite dimensional dynamics systems in mechanics and physics. Berlin, Heidelberg, New York: Springer 1988

16. Yudovich, V. I.: Example of the generation of a secondary stationary or periodic flow when there is loss of stability of the laminar flow of a viscous incompressible fluid. J. Appl. Math. Mech. 29 (1965)

Communicated by J. L. Lebowitz 